\title{
Educación y tecnologías de la información y la comunicación ¿es posible valorar la diversidad en el marco de la tendencia homogeneizadora?
}

\author{
GEORGINA VIVANCO \\ Universidad de Santiago de Chile, \\ Santiago, Chile
}

\section{RESUMEN}

Este artículo reflexiona críticamente sobre la incorporación de las tecnologías de la información y la comunicación (TIC) en la educación, cuestionando la posibilidad de valorar la diversidad en la actual tendencia homogeneizadora de las políticas educacionales. A partir de una investigación bibliográfica, se relevan los contextos de las políticas y programas de incorporación de las TIC en la educación y las lógicas que las sustentan. Se propone contribuir con una perspectiva cultural para abordar el aprendizaje escolar con tecnologías digitales, dentro de marcos de igualdad democrática pero con orientación a la diversidad cultural. Se parte explorando el escenario de globalización y cómo este condiciona la racionalidad que subyace a las políticas públicas en educación. También se reflexiona sobre cómo las TIC han dejado de ser un medio, para convertirse en un ecosistema comunicativo que favorece nuevas subjetividades $\mathrm{y}$ formas de estar en el mundo. Finalmente, se enfatiza la necesidad de profundizar la dimensión cultural en la incorporación de las tecnologías en la educación y su articulación transversal con las dimensiones pedagógicas y de inclusión social.

PALABRAS CLAVE

tecnologías y educación; políticas educacionales; tecnologías de la información y de la comunicación; interacción digital comunicativa. 


\section{EDUCATION AND INFORMATION AND COMMUNICATION TECHNOLOGIES: IS IT POSSIBLE TO ASSESS THE DIVERSITY WITHIN THE HOMOGENIZER TREND?}

\section{ABSTRACT}

This article reflects critically on the incorporation of information and communication technologies (ICT) in education, questioning the possibility of valuing diversity in the homogenizing tendency of current educational policies. Contexts, policies and programs which incorporate ICTs in education are surveyed as well as the logic that underpins them. It aims to contribute to a cultural perspective which addresses student learning with digital technologies within frameworks of democratic equality but oriented toward cultural diversity. We explore the context of globalization, and how this affects the underlying rationale for public policies in education and reflect on how ICTs are no longer a medium but instead have created a communication ecosystem that favors new subjectivities and ways of existing in the world. Finally, it emphasizes the need to deepen the cultural dimension in the incorporation of technology in education and its articulation within the transverse dimensions of pedagogical and social inclusion.

\section{KEYWORDS}

technology and education; educational policies; information and communication technology; digital communicative interaction.

\section{EDUCAÇÃO E TECNOLOGIAS DA INFORMAÇÃO E COMUNICAÇÃO: É POSSÍVEL VALORIZAR A DIVERSIDADE NO MARCO DA TENDÊNCIA HOMOGENEIZADORA?}

\section{RESUMO}

Este artigo reflete criticamente sobre a incorporação das tecnologias de informação e comunicação (TIC) na educação, questionando a possibilidade de valorizar a diversidade na atual tendência homogeneizadora das políticas educacionais. Com base em uma pesquisa bibliográfica, relevam-se os contextos das políticas e programas de incorporação das TIC na educação e as lógicas que as sustentam. Propõe-se contribuir com uma perspectiva cultural para abordar a aprendizagem escolar com tecnologias digitais, dentro de marcos de igualdade democrática, mas orientado à diversidade cultural. Parte-se explorando o cenário de globalização e como este condiciona a racionalidade subjacente às políticas públicas em educação. Também é feita uma reflexão sobre como as TIC deixaram de ser um meio para se converterem em um ecossistema comunicativo que favorece novas subjetividades e formas de estar no mundo. Finalmente, enfatiza-se a necessidade de aprofundar a dimensão cultural na incorporação das tecnologias na educação e sua articulação transversal com as dimensões pedagógicas e de inclusão social.

PALAVRAS-CHAVE

tecnologias e educação; políticas educacionais; tecnologias de informação e comunicação; interação digital comunicativa. 


\section{INTRODUCCIÓN}

Este artículo propone explorar la incorporación de las tecnologías de la información y la comunicación (TIC) en la educación en los actuales escenarios marcados por la globalización, la cual emerge y se impone en su doble naturaleza, como posibilidad y como perversidad (Santos, 2004 apud Martín-Barbero, 2008), como unificadora y a la vez diversificadora, o bien homogeneizadora pero también revitalizadora y generadora de resistencias (Morin, 2011). En fin, se evidencia un marco complejo, por lo que se torna relevante entender las lógicas que fundamentan los discursos, las reformas y las acciones educacionales que pretenden regular los patrones sociales.

Se analiza el predominio de una racionalidad tecnicista, amparada en el universalismo abstracto que orienta una perspectiva particular de calidad. Perspectiva que establece un vínculo directo entre escolarización y competitividad internacional de las economías nacionales. Es en esta perspectiva que se originan reformas educativas que orientan las políticas educacionales y los sistemas de evaluación de dichas políticas (Garcia, 2010).

El universalismo abstracto que sirve de sustrato a las políticas y reformas educacionales parte de categorías que se abstraen de toda determinación espaciotemporal (universalismo de los enunciados). Pero, a su vez, se expresa el universalismo del sujeto de enunciación, es decir, la imposición de una visión y perspectiva particular de mundo: la eurocentrista (Grosfoguel, 2008). Ahora bien, si en un sentido el ser excluidos e ignorados como visión y perspectiva puede ser un factor valorado como negativo, por otro lado ello ha permitido un resultado paradojal: que estas culturas del sur se hayan conservado y hayan desarrollado atributos que faltan al occidente o al norte (Martucceli, 2010).

A partir de ahí se introduce la temática de las TIC en la educación en los actuales contextos globalizados. Las tecnologías digitales irrumpen en la educación, como en las otras esferas de la sociedad, pero con la particularidad que no fueron concebidas ni ideadas dentro y para el ambiente educacional, es decir, no se gestan ni se introducen a partir de auténticas necesidades educativas. Es así que frecuentemente se genera una mistificación de las bondades de las TIC, y sus posibles contribuciones a los contextos de aprendizajes. En este artículo se propone una mirada crítica, distinguiendo sus potencialidades, pero también sus limitaciones. Para guiar esta perspectiva se proponen tres dimensiones que sintetizan las intencionalidades que orientan la incorporación de las TIC: la primera responde a intencionalidades pedagógicas, la segunda a intencionalidades de inclusión social y, por último, las más próximas a intencionalidades culturales. A veces se implementan de forma dicotomizada, y otras de una forma más relacional y solapada. Pero en general predominan las dos primeras dimensiones, estando presente la dimensión cultural con poca frecuencia.

Se rescata el concepto de externalidad de Dussel (2004) como alternativa para articular las aspiraciones multiculturales y el universalismo democrático de Benhabib (2006), con el universalismo concreto o pluriverso de las culturas periféricas de nuestra región que propone aquel autor. Con esta trama de fondo, 
se propone la dimensión cultural de incorporación de las TIC en la educación como alternativa para lograr un punto de equilibrio entre el uso predominante caracterizado por el aprendizaje dentro de marcos de igualdad democrática (tendencia homogeneizadora) y el uso para la diversidad cultural. Se entiende que este equilibrio es una alternativa para valorar las diferencias dentro de un marco de igualdad de derechos.

A partir de esta entrada, y reivindicación, de la dimensión cultural de las TIC, se exploran estas nuevas formas de estar en el mundo y las subjetividades que se crean y recrean en este nuevo ecosistema comunicacional. Contexto para nada irrelevante cuando de problemas educacionales se trata, ya que involucra nuevas configuraciones mentales, sensoriales y emotivas, que han transformado las formas de interactuar, de relacionarse y de comunicarse, implicando en identidades que no se configuran como una sumatoria de identidades que conviven, sino que se alteran mutuamente a través de una interacción horizontal. Este nuevo ecosistema comunicacional puede ser visualizado como un espacio de homogeneización y de voluntad de borradura de las diferencias, pero también puede ser visualizado como un espacio donde las diferencias logran escapar y manifestarse. Así como la globalización, las TIC encarnan una doble naturaleza, y el mismo instrumento que se incorpora con fines de homogeneización puede ser invocando en pro del derecho a la diferencia.

Queda enunciado así el desafío que conlleva la incorporación del enfoque cultural de las TIC en la educación, desafío complejo, pero sumamente relevante considerando que las TIC han dejado de ser un medio, para convertirse en un ecosistema comunicativo que está favoreciendo nuevas subjetividades. Esta dimensión cultural puede servir de sustento transversal, para orientarse intencionadamente, y potencializar los logros en las otras dimensiones, la pedagógica y la de inclusión social.

\section{DESDE LA GLOBALIZACIÓN: HOMOGENEIZACIÓN Y HETEROGENEIZACIÓN}

Morin (2011) señala que la globalización, a la vez una y plural, conoce una crisis propia, que acerca y disgrega, unifica y separa. Por su parte, Santos (2004 apud Martín-Barbero, 2008) lo expresa como la simultaneidad entre perversidad y posibilidad. La perversidad resultado del proceso avasallador del mercado, que favorece la creación de una cultura de masas y de este modo cierta uniformación cultural, comprometiendo, de esta forma, la diversidad y fragilizando lo nacional y lo local. Pero la globalización también puede ser vista como un conjunto de oportunidades, ya que permite un mayor entrelazamiento de pueblos y culturas, favorece la emergencia de reivindicaciones de identidades culturales y étnicas como expresiones de resistencia (Marín, 2010), es decir, cosmovisiones opuestas a la hegemonía del racionalismo occidental.

La globalización tanto divide como une; divide mientras une $-y$ las causas de la división son idénticas a las que promueven la uniformidad del globo [...]. Lo 
que para algunos parece globalización, para muchos otros significa localización; lo que para algunos es señal de libertad, para muchos otros es un destino indeseado y cruel. (Bauman, 1999, p. 8)

Morin (2011) sintetiza el escenario generado por la globalización como el antagonismo y concurrencia de tres procesos culturales: la homogeneización, las resistencias y revitalización de culturas autóctonas y el mestizaje cultural. En línea con estas afirmaciones Benhabib (2006) reflexiona sobre las nuevas dinámicas sociales que resultan de la tensión entre integración global junto con desintegración sociocultural, emergiendo una tensión entre la homogeneización social, cultural y económica por un lado, y por el otro la resistencia, subversión y protesta a esta homogeneización, a la que llama extraña multiplicidad.

Morin (2011, p. 27) al reflexionar sobre la crisis del desarrollo, afirma que es ocasionada por la mundialización de la trina: globalización, occidentalización y desarrollo. Esta trina responde a una concepción tecnoeconómica del desarrollo, y utiliza fundamentalmente el cálculo como instrumento de conocimiento, que ignora todo aquello que no puede calcularse ni medirse, es decir, "el tejido mismo de nuestras vidas". Morin (idem, p. 17) le atribuye al desarrollo tecnoeconómico ser el causante de un subdesarrollo intelectual por la falta de percepción global; de un subdesarrollo psíquico por responder básicamente a la lógica económica; y de un subdesarrollo moral por basarse en un egocentrismo. Concluye afirmando que "la idea de desarrollo es una idea subdesarrollada”.

\section{POLÍTICAS HOMOGENEIZADORAS}

Es importante entender las lógicas que subyacen al proceso de globalización en las reformas actuales, las cuales se establecen como prácticas globales que se proponen cambios en los patrones de regulación social. Para ello, incorporan racionalidades y "prácticas de exclusión e inclusión que resultan en clasificaciones o distinciones que forman un posible horizonte ético del individuo consigo mismo y con los otros" (Garcia, 2010, p. 446). En este contexto surgen varios interrogantes, uno de los cuales podría ser cómo neutralizar, en lo posible, esa racionalidad orientada a un objetivo en la relación de poder (Foucault, 1988), que genera medios - por ejemplo, las reformas educacionales- para implementar o mantener el poder de forma efectiva, actuando, de esta forma, como modelo de acción sobre posibles acciones. Otro interrogante podría ser cómo superar estas lógicas que representan un universalismo abstracto y generar alternativas con otro tipo de conciencia, que entienda, por ejemplo, que "la unidad humana engendra la diversidad humana y la diversidad humana mantiene la unidad humana” (Morin, 2011, p. 12).

Aparece un nuevo ideal tecnicista en la base de las reformas del Estado y de los principios más generales que pautan las reformas contemporáneas, y está relacionado con la intención de definir un cierto orden, que permita lograr resultados y eficiencia con costos mínimos y previsibles. Por otro lado, resultado de esta intención, se produce una crisis en la identidad plena entre el Estado y la sociedad civil característica de los Estados de Bienestar y se busca generar un 
Estado ampliado con una dinámica descentralizada, apoyándose en formas de poder individualizador y con una aparente devolución de los poderes reguladores del Estado para "abajo", delegando la responsabilidad de la gestión de los recursos a instancias intermediarias (Garcia, 2010).

Ruiz (2011) señala que uno de los efectos de la globalización en el ámbito internacional sería el establecimiento de ciertos marcos para establecer gobierno sin gobierno, destacándose instituciones internacionales como el Fondo Monetario Internacional (FMI), la Organización para la Cooperación y el Desarrollo Económico (OCDE), el G-7, el Banco Mundial y la Unión Europea; y señala cómo algunas instituciones, como es el caso de la OCDE, se están manifestando en el ámbito educativo con un protagonismo significativo. Muchos investigadores coinciden en que en el actual momento de globalización, este organismo ha ocupado un espacio en la política educativa nacional e internacional por derecho propio (Henry et al., 2001 apud Ruiz, 2011).

Existe, por lo tanto, una lógica que representa el discurso y las expectativas de estas instituciones internacionales. Instituciones como la OCDE analizan las problemáticas educativas desde un fundamentalismo económico y desde una dimensión esencialmente técnica. Los criterios internacionales utilizados para evaluar la calidad de los sistemas nacionales de educación, por ejemplo en el Programme for International Student Assessment (PISA), parten de valorar, fundamentalmente, la capacidad de formar estudiantes que favorezcan la competitividad internacional de las economías nacionales (Ruiz, 2011). Esto explicaría la relevancia que adquieren los resultados de dichos estudios en los países participantes, ya que reflejarían, en alguna medida, la eficacia de sus sistemas educativos y la calidad de su capital humano dentro de este prisma o alineados con esta proyección de futuro. Permitiendo comprender de este modo el "interés político en asegurar la cobertura y el acceso universal a la educación, así como el aprendizaje efectivo de todos los alumnos de los nuevos contenidos curriculares" (Ferrer; Arregui, 2003, p. 5).

Es decir, hay una intención de establecer vínculos entre "escolarización, empleo, productividad y mercado; mejorar los resultados de aprendizajes relacionados a las competencias y destrezas que requiere el mundo laboral" (idem, p. 6), y es esta relación la que explica que algunas agencias de cooperación internacional condicionen sus préstamos a la educación, al desarrollo de sistemas nacionales de medición que permitieran medir los resultados de esas millonarias inversiones en reformas educativas dentro de este marco epistémico (idem).

A partir de estas racionalidades se generan las reformas, que son "prácticas globales que pretenden crear cambios en los patrones de regulación social y en los regímenes éticos que capacitan a los sujetos para las relaciones sociales" (Garcia, 2010, p. 446). Esta autora señala que las reformas funcionan como un conjunto de principios y directrices que deben guiar la acción educacional y que son esfuerzos intencionados para favorecer que se generen ciertas racionalidades en las disposiciones y conciencias de los sujetos. Es decir, las reformas educacionales, y las políticas educacionales, buscan generar "cambios en las capacidades interiores, cognitivas e instrumentales de los sujetos, y que esos cambios coincidan con los objetivos de la administración social de las poblaciones" (idem, ibidem). 
De esta forma, las políticas educacionales, que forman parte de la llamada modernización, pretenden tornar el mundo legible y transparente, anulando, para esto, las posibles contingencias, donde "modernizar significa, entre otras cosas, tornar el mundo habitado receptivo a la administración supracomunitaria, estatal; y esa tarea requiere, como condición necesaria, tornar el mundo transparente y legible para los poderes administrativos" (Bauman, 1999, p. 34).

Las reformas educacionales que orientan las políticas públicas, además de regirse por lógicas que se derivan de las regulaciones determinadas por las instituciones internacionales, se apoyan en el cálculo de la eficiencia y el costo (Morin, 2011), el cual ignora el tejido mismo de nuestras vidas, los contextos humanos y culturales, las singularidades y presenta el modelo occidental como el arquetipo universal.

Sin embargo, como - por suerte- no todo es previsible, y gracias a esta maravillosa característica compleja de los fenómenos, son justamente esos estudios del estilo del PISA de la OCDE, cuyas bases epistemológicas se podrían advertir como perversas, en el sentido que pretenden perpetuar y agravar las profundas desigualdades sociales con un discurso de un aparente principio igualitario, los que están generando debates y cuestionamientos que van más allá de los límites de los propios estudios. Y sería en este contexto que se pretende realizar esta reflexión. Es así que surgen debates como, por ejemplo, el de las categorías generadas en estos estudios, las cuales están orientadas a claros objetivos de lógicas instrumentalistas económicas. Ruiz (2011, p. 3) nos advierte cómo en PISA se incorpora una definición de equidad sumamente reducida, considerando "parámetros concretos y puntuales como el acceso a las instituciones educativas", y desconsiderando cuestiones socioculturales.

\section{UNIVERSALISMO ABSTRACTO}

Estos estudios, los cuales sirven de guía para las políticas educacionales, se basan en el universalismo abstracto, que no es otra cosa que categorías vaciadas de todo contexto. Es decir, se hace uso público de la razón creando un lugar formal que trata de representar un nolugar, tratando de eliminar cualquier vestigio del lugar del cual procedemos como sujetos empíricos. Grosfoguel (2008) lo expresa como el universalismo occidental -de los enunciados y el del sujeto de enunciación-. Este universalismo expresado en los estudios internacionales de educación pretende generar un conocimiento eterno que se abstrae de toda determinación espaciotemporal utilizando para esto categorías que según esta comprensión representan a cualquier situación por el solo hecho de descontextualizarla. Este sería el universalismo en cuanto a los enunciados. Pero en los estudios también se manifiesta el segundo sentido de universalismo abstracto, el del sujeto de enunciación, el de la ego-política del conocimiento, ya que estos estudios, en general, son generados desde una visión occidental eurocentrista, sin incorporar los contextos latinoamericanos. En palabras de Grosfoquel (idem, p. 202), 
Se trata entonces de una filosofía donde el sujeto epistémico no tiene sexualidad, género, etnicidad, raza, clase, espiritualidad, lengua, ni localización epistémica en ninguna relación de poder, y produce la verdad desde un monólogo interior consigo mismo, sin relación con nadie fuera de sí. Es decir, se trata de una filosofía sorda, sin rostro y sin fuerza de gravedad. El sujeto sin rostro flota por los cielos sin ser determinado por nada ni por nadie.

Grosfoguel afirma que si se crea una propuesta global que se construye desde un territorio y cuerpo particular en exclusión de los otros, el mismo procedimiento le otorga a la propuesta un carácter inherente imperialista/colonial. Y, por otro lado, señala que si la única "tradición de pensamiento con dicha capacidad de universalidad y de acceso a la verdad es la occidental, entonces no hay universalismo abstracto sin racismo epistémico" (idem, p. 208). Concluye que el racismo epistemológico es intrínseco al "universalismo abstracto" occidental, que encubre a quien habla y el lugar desde donde habla.

Pero las experiencias fueron hechas y los resultados, hoy lo podemos constatar, no han sido muy alentadores. Ese afán por explicar los fenómenos objetivando el conocimiento alejó al hombre del real conocimiento, rotulando, excluyendo y sentenciando todo aquello que no fuese regido por leyes objetivas. Si nuestro mundo debe ser comprendido desde una visión determinista y de sistemas estables, no tendría nada en común con el mundo real que nos rodea, acaso sería un mundo estático y predecible, pero no estaríamos en él para realizar nuestras predicciones (Prigogine, 1996).

Es indispensable plantearse la reflexión, estudio y propuestas para tratar los fenómenos educativos desde prismas que representen tanto nuestras realidades como las necesidades de nuestra región. Según Martucceli (2010), el occidente perdió el monopolio sobre la modernidad. Una serie de factores como la globalización, la consolidación de importantes sectores de clase media en los países emergentes, la producción científica y tecnológica en los países del Sur, la transformación de las relaciones de poder entre Estados nos proyectan a un mundo en que la modernidad ha dejado de ser el monopolio de occidente. $\mathrm{El}$ autor se hace una serie de preguntas que nos podrían orientar en la definición de intencionalidad con colores propios:

¿Por qué interpretar las experiencias latinoamericanas como figuras incompletas o anómalas? ¿Por qué negar sus diferencias comparándolas con una experiencia de la modernidad erigida en modelo universal? ¿No es acaso necesario abandonar la idea de una modernidad única en beneficio de una diversidad de modernidades? ¿No es ésta la única vía que nos permitirá, ipor fin!, romper verdaderamente con el occidentalo-centrismo? (idem, p. 23)

Martucceli resalta la modernidad-colonialidad como dos caras de una misma moneda, y afirma que el norte (occidente) sólo entiende una de ellas, y solamente desde nuestros países del sur se puede comprender la doble densidad de este proceso. Continúa afirmando que para entenderlo hay que verlo desde un conocimiento "geopolítico" que privilegie la problemática de las historias de las fronteras, ya que 
nuestras regiones se caracterizan por la mezcla en contraposición con la pureza y separación de occidente, esta mezcla se funde en la modernidad, desfigurándola y hasta la podríamos interpretar como el inicio de modernidad o la continuación de la descolonización. Termina este autor afirmando que "el individuo en el Sur sólo existe desde la diferencia" (idem, p. 29).

\section{TIC Y EDUCACIÓN EN ESTOS CONTEXTOS GLOBALIZADOS}

A la par de los contextos globalizados, la sociedad contemporánea ha sido afectada/beneficiada por nuevos escenarios, formas de relación, aprendizajes y vivencias, productos de la inserción/irrupción de las TIC en nuestros contextos de vida. Resulta difícil limitar el papel de las nuevas tecnologías a meros instrumentos o medios, ya que deben ser consideradas más bien como un nuevo ambiente que incluye todo dentro de sí (PNUD, 2006). Martín-Barbero (2008) sostiene que las TIC son la base de un nuevo entorno o ecosistema comunicativo que está configurando nuestros modos de habitar el mundo y las formas mismas del lazo social.

Las TIC transformaron y siguen transformando de manera significativa nuestro lenguaje, nuestras formas de pensar, aprender, comunicarnos, trabajar y decidir. También cuestionan las nociones de espacio y de tiempo resultado de un nuevo sistema técnico a escala planetaria que transforma el uso del tiempo al producir la convergencia y simultaneidad de los momentos en todo el mundo (idem), remplazan nuestras habituales formas de pensamiento lineal por uno no lineal y multitarea. En fin, se caracterizan por su capacidad de modificar el curso $\mathrm{y}$ funcionamiento de las relaciones y las actividades humanas. Es justamente esa potencia disruptiva y sistémica lo que torna complejo, y al mismo tiempo decisivo, el proceso de la integración de las TIC en la educación.

Si bien esta irrupción ha sido fuerte y transversal en múltiples contextos -educacional, comercial, familiar, etc.--, se constata que las nuevas tecnologías no fueron concebidas ni imaginadas para la educación; no aparecen naturalmente en los sistemas educacionales, y raramente su introducción respondió a intencionalidades educativas. Es decir, el movimiento de su incorporación ha sido más bien desde el exterior hacia el interior del contexto educacional (Dussel; Quevedo, 2010). Ello ha inhibido en general el desarrollo de una fuerza en sentido contrario, de demandas desde el propio sistema educativo, capaz de favorecer la posibilidad de regular/complementar/colaborar con esta incorporación tecnológica. Como resultado no se ha podido llegar a establecer una relación dialógica, sino más bien de imposición o sometimiento. Este puede ser justamente uno de los problemas claves de la relación entre educación y TIC; es decir, la dificultad para implantar en la educación elementos que le son extraños, que no surgen ni se desarrollan en su seno y, por tanto, que no se operan en ellos de manera natural.

Frecuentemente la incorporación de las TIC está asociada a una mistificación, como si ellas fueran automáticamente portadoras de nuevos contextos y oportunidades de aprendizaje. En verdad, las TIC "per se" no necesariamente favorecen un aprendizaje real o novedoso. A veces se trata solamente de un cambio 
de escenario, donde la escenografía adquiere tanta importancia que los aspectos más simples y esenciales del aprendizaje resultan diluidos, disimulados y -se podría arriesgar a decir- excluidos, en un entorno caracterizado esencialmente por un bombardeo de estímulos.

Las nuevas tecnologías están colaborando con un crecimiento de la circulación de saberes y de información. Sin embargo, también se constata que esta curva creciente es acompañada por otra curva, también creciente, asociada a una sensación de saturación ante tanta información y la imposibilidad de realizar las conexiones necesarias y pertinentes para entender su sentido, significarla, criticarla, discutirla e interiorizarla. Se produce así una distancia entre lo que sabemos y lo que realmente comprendemos, no consiguiendo integrar los conocimientos en la conducta de nuestras vidas. Como se pregunta Eliot (1934 apud Morin, 2002, p. 17): “¿Dónde está el conocimiento que perdemos en la información? ¿Dónde está la sabiduría que perdemos en el conocimiento?”.

Resulta problemático, por lo tanto, validar la incorporación de las TIC en los contextos educacionales sin discutir y evaluar las mediaciones necesarias en línea con las intenciones educacionales. Es necesario visualizar sus ventajas, pero también comprender qué se pierde o qué se sacrifica. Esto es, hacer emerger la dimensión educacional de la incorporación de las TIC en los diferentes contextos, lo que puede ser logrado a través de una clara exposición de sus potencialidades y de un abordaje intencionado de sus limitaciones. El sistema educativo, al ser la principal institución del Estado con aptitud para disociar las diferencias en los orígenes sociales, está llamado a cumplir un rol fundamental en este sentido; es el "medio privilegiado para asegurar un dinamismo productivo con equidad social, tender puentes de comunicación en sociedades multiculturales, y fortalecer democracias basadas en el ejercicio ampliado y sin exclusiones de la ciudadanía” (Sunkel; Trucco, 2010).

Se han desarrollado, en la región y en el mundo, una gran variedad de modelos e investigaciones que apuntan a visualizar la influencia de las TIC en la educación. Pero aún los resultados no son unívocos ni concluyentes, lo que señala la necesidad -ampliamente aceptada- de continuar la investigación, el estudio de experiencias y el levantamiento de evidencias empíricas (Claro, 2010). Es en este sentido que resulta relevante explicitar una intencionalidad educacional en este proceso de incorporación. Hay muchos avances en este sentido y las investigaciones lo confirman. Sin embargo, parecería que los resultados de la integración de las TIC en la educación no están a la misma altura que las expectativas que se crearon. Los estudios denotan dificultades de infraestructura, o de formación de los profesores en la utilización de los recursos, o de metodologías que no acompañan las características de los nuevos escenarios de los estudiantes fuera de los contextos de educación formal.

En las propuestas de incorporación de las TIC en educación, se observa que básicamente responden a tres enfoques o intencionalidades: a intencionalidades pedagógicas, a intencionalidades orientadas a la inclusión social -que puede lograrse a través de la inclusión digital- y, por último, las más próximas a aspectos culturales. Estas dimensiones pueden ser abordadas de forma dicotomizada, pero también pueden ser abordadas de forma multidimensional y relacionada. A veces 
se opta por enfoques que relevan principalmente los aspectos pedagógicos, pero que a su vez pueden traer resultados alentadores en la inclusión digital y, por ende, en la inclusión social. De forma inversa, se podría establecer la relación contraria, iniciar con motivaciones de inclusión digital-social que derivan en beneficios de aprendizajes de los estudiantes. Otra posible dimensión, la cultural, es tratada de una forma transversal, pero son pocas las iniciativas que declaran explícitamente este enfoque como dimensión central.

\section{DESDE LA EXTERIORIDAD: LAS CULTURAS PERIFÉRICAS COMO PUNTO DE PARTIDA PARA INTERACCIONES DIGITALES DELIBERATIVAS}

Se propone reflexionar sobre posibles alternativas al escenario de una sociedad globalizada, con una irrupción de las TIC en todas las esferas Para esto se busca la confluencia de algunos conceptos de Enrique Dussel a partir de las posibilidades que se generan desde la externalidad de la modernidad, con algunas nociones de Seyla Benhabib en relación a las prácticas deliberativas. De alguna forma se trata de articular las aspiraciones multiculturales y el universalismo democrático al que adhiere Benhabib, con el universalismo concreto o pluriverso de las culturas periféricas de nuestra región que propone Dussel.

Dusell (1994 apud Grosfoguel, 2008) propone un universalismo concreto y pluriverso en el sentido de trascender la versión eurocéntrica de la modernidad con la propuesta de transmodernidad, la cual, más que culminar la modernidad, propone culminar el poscolonialismo. El autor instiga así a generar propuestas críticas descolonizadoras de la forma liberal occidental de democracia desde las más diversas localizaciones culturales, permitiendo de esta forma la resignificación del concepto de democracia desde un prisma transmoderno.

Las construcciones categoriales con visiones eurocentristas reflejan su intención de perpetuar las relaciones asimétricas y coloniales en los planos económicos, políticos y culturales; como también la intención de perpetuar el no-diálogo de culturas y pueblos en su propia construcción. Y es en lo que se excluye o lo que queda en la marginalidad, lo que permite la posibilidad de otra realidad, es justamente allí donde aparece con mayor fuerza la potencialidad de otras posibles realidades. Dussel (2004) afirma que esas otras culturas han sido colonizadas, pero en la mejor estructura de sus valores han sido excluidas, despreciadas, negadas, ignoradas más que aniquiladas. Y es justamente ese desprecio, el que las condujo a la exclusión, el que ha permitido que aún sobrevivan: esa sería una posible brecha.

[...] esa cultura periférica oprimida por la cultura imperial debe ser el punto de partida del diálogo intercultural [...]. La cultura de la pobreza cultural, lejos de ser una cultura menor, es el centro más incontaminado e irradiativo de la resistencia del oprimido contra el opresor [...]. Para crear algo nuevo ha de tenerse una palabra nueva que irrumpe a partir de la exterioridad. Esta exterioridad es el propio pueblo que, aunque oprimido por el sistema, es lo más extraño a él. (idem, p. 7) 
Colocarse desde esta externalidad que propone Dussel puede ser pertinente cuando se piensa la interculturalidad en la educación para nuestra región. Este autor hace una afirmación muy interesante sobre la posibilidad de diálogo entre culturas: "para intentar el diálogo intercultural habría que comenzar por hacer un diagnóstico de los contenidos últimos de las narrativas míticas, de los supuestos ontológicos y de la estructura ético-política de cada una de ellas" (idem, p. 3).

No se puede establecer un diálogo desde el universalismo abstracto de la modernidad, primero, porque es un universalismo moderno eurocentrista que lejos de dialogar, impone, y,segundo, porque no todas las partes son modernas, y menos aún posmodernas. Pero este mismo motivo que impide el diálogo, es el que permitiría irrumpir desde la externalidad, y proponer desde ese lugar sus propias experiencias culturales, y manifestarse con capacidad de responder con soluciones absolutamente imposibles para la cultura moderna (idem). Según Dussel, un diálogo intercultural pluriverso debería asumir los momentos positivos de la modernidad, pero evaluados con criterios distintos desde otras culturas milenarias. Es este rescate, de la modernidad contextualizada en nuestras realidades periféricas (transmodernidad) que se quiere articular con el constructivismo social de Benhabib.

Benhabib (2006) propone el constructivismo social como explicación abarcadora de las diferencias culturales, y como contrapropuesta a los intentos de la teoría política normativa de reificar a los grupos culturales y sus luchas por el reconocimiento. La autora reivindica esta opción y critica el esencialismo cultural, desde una visión narrativa de las acciones y la cultura que las caracteriza. Posición epistémica que la diferencia de los autores del multiculturalismo mosaico, los cuales entienden la cultura y los grupos humanos como totalidades bien delineadas e identificables que coexisten con fronteras claras, análogas a un mosaico. E1 multiculturalismo mosaico esencializa la idea de cultura como propiedad de un grupo étnico o de una raza, enfatiza la homogeneidad interna de las culturas y trata a las culturas como insignias de identidad grupal.

Por su parte, el constructivismo supone que el mundo es una construcción, de la misma manera que las pautas que lo rigen, las cuales no son fijas ni inmutables. La cultura emerge de efectos narrativos y se presenta a sí misma a través de relatos controvertidos debido a dos razones: por la doble hermenéutica de los relatos y la postura valorativa de los mismos. $Y$ es en estos efectos narrativos que acontece la hibrides, cuestión que se da con independencia de las actitudes de la diferencia en cuestión, donde lo moderno y lo tradicional interactúan con independencia y se va generando una tercera cuestión. Benhabib reivindica el momento político de la interacción y de la diferencia de la siguiente forma:

[...] deberíamos considerar las culturas humanas como constantes creaciones, recreaciones y negociaciones de fronteras imaginarias entre nosotros y el/los otros. El otro siempre está también dentro nuestro y es uno con nosotros. Un sí mismo es un sí mismo sólo porque se distingue de un otro real, o más que nada imaginario. (idem, p. 33) 
Para Benhabib (idem) la cultura se crea a partir del diálogo complejo con otras culturas, el diálogo con el otro es intrínseco antes que extrínseco a la cultura en sí, y entiende que la lucha por el reconocimiento no es dicotómica o excluyente de la inclusión democrática. Afirma que los movimientos culturalistas pueden ser críticos y subversivos en la medida en que sus motivaciones no sean conservacionistas. Importa mucho si defendemos las exigencias culturalistas porque se quiere preservar las culturas minoritarias dentro del Estado democrático liberal, o porque deseamos ampliar el círculo de inclusión democrática. La autora adhiere a una sociedad democrática deliberativa que permita la controversia cultural dentro de la esfera pública, donde se pueda lograr hacer realidad las oportunidades para la máxima autoadscripción cultural y la justicia intergrupal colectiva. Sostiene que un enfoque modernista de las culturas -como creaciones de sentido controvertidas-y un enfoque universalista de democracia deliberativa se complementan entre sí.

\section{TIC EN SU DIMENSIÓN CULTURAL}

Según Benhabid (idem, p. 45) "nacemos en redes de interlocución o redes narrativas, desde relatos familiares y de género hasta relatos lingüísticos y los grandes relatos de la identidad colectiva. Somos conscientes de quiénes somos aprendiendo a ser socios conversacionales en estos relatos". Este planteamiento es muy pertinente para retornar a la temática de las TIC en la educación, pero desde la intención de explicitar su dimensión cultural y sus posibilidades como instrumento cultural en el tejido de estas redes narrativas que son las que configuran nuestra cultura.

Las TIC no siempre pueden ser consideradas objetos culturales, de hecho su incorporación más usual en los contextos de aprendizaje es como herramienta tecnológica. Pero en este trabajo se pretende realzar su esencia cultural y cómo dicha esencia desencadena una cierta tensión entre las nuevas subjetividades creadas a través de su uso, las que se caracterizan por su horizontalidad, colaboración y cooperación, y el orden social al interior de los ambientes escolares, caracterizados por una dinámica bien contrapuesta a esta horizontalidad.

Con la idea de distinguir la dimensión cultural en la incorporación de las TIC en contextos educacionales, se rescata a Castoriadis (2001), quien propone establecer una distinción entre la cultura, en el sentido estricto del término, y la dimensión puramente funcional de la vida social. Para este autor "la cultura es el dominio del imaginario en el sentido estricto, el dominio poiético, lo que en una sociedad llega más allá de lo que es solamente instrumental” (idem, p. 98). No hay sociedad sin cultura, así como las sociedades no pueden ser reducidas a lo estrictamente funcional o instrumental. Para establecer una distinción entre lo que llama poiético y lo funcional, el autor continua afirmando que esta distinción "no se encuentra en las cosas, sino que se encuentra en la relación entre cómo se hacen las cosas y su finalidad. Es así que la poiética y lo funcional no tienen el mismo ritmo ni el mismo sentido" (idem, ibidem).

Este sentido poiético, en contraste con el sentido funcional, puede representar un sustento para distinguir la utilización de las TIC en su dimensión pedagógica de su dimensión cultural. No se propone establecer una relación de exclusión o 
dicotomizar ambas dimensiones, sino entenderlas como dos dimensiones que pueden dialogar y pueden complementarse. Sin embargo, optar por una sola de ellas e ignorar la otra implica una reducción de las potencialidades de las TIC. Aquí hay una afinidad con la reflexión de Castells cuando se refiere a la transcendencia de internet: no interesa cuánto se usa, sino por qué y para qué se usa (Castells et al., 2007).

Es importante distinguir estas dimensiones del uso de las TIC y los énfasis que se realizan en su incorporación. Cuando optamos, por ejemplo, por un sentido funcional a los aprendizajes sin reflexionar en un para qué, podría resultar, también, funcional a la homogeneización en estos contextos globalizados, para lo cual las TIC se presentan como una herramienta idónea. En efecto, el uso de las TIC puede implicar tanto homogeneización como hibridación absoluta, que no es otra cosa que homogeneización, ya que podría significar una negación de la identidad y transformarse finalmente en una herramienta para una nueva homogeneización.

Sin embargo, cuando se opta por relevar el sentido cultural de las TIC se está enfatizando el significado controvertido de las tecnologías, el menos evidente, el más difuso, y muy probablemente el menos deseado desde las intenciones de las culturas mayoritarias en la relación de poder. En este punto, el concepto de externalidad de Dussel es pertinente, que se refiere a actuar desde la externalidad impuesta por la cultura dominante. Es así que el mismo instrumento que se incorpora para homogenizar puede ser rescatado desde la externalidad para reivindicar el derecho a la diferencia. Porque al igual que la globalización, las TIC unen y dividen, son el exponente de esa paradoja y encarnan esta característica de los nuevos tiempos.

Aparece entonces la necesidad de demarcar un punto de equilibrio, entre el uso para el aprendizaje dentro de marcos de igualdad democrática (tendencia homogeneizadora) y el uso para la diversidad cultural, cuestión extremadamente compleja pero absolutamente necesaria. Lograr este equilibrio significaría valorar las diferencias dentro de un marco de iguales derechos, y podría ser visualizado como un horizonte a ser alcanzado. Partiendo de reconocer esta complejidad, se busca explicitar la necesidad de definir intencionalidades desde los contextos educacionales hacia estas fricciones, y no caer en la ilusión a la que alude Zizek del capitalismo del ciberespacio: la ilusión de un "medio de intercambio completamente transparente, etéreo, en el que desaparecen hasta los últimos rastros de la inercia material" (Zizek, 1998, p. 10). Es decir, si bien valorar las diferencias es un escenario complejo e hipotético, casi que ideal, debe ser intencionado socialmente como un escenario deseable.

La dimensión cultural de la incorporación de las TIC puede servir de sustento para proyectarse de forma intencionada en su dimensión pedagógica o educacional. Se parte de la tesis que las TIC entraron en nuestras vidas para quedarse, y su inserción está generando otras formas de estar en el mundo. Están provocando una modificación sustancial del sujeto, la cultura, las relaciones sociales y el acceso al saber. Han surgido diversas formas de aprender, las cuales se han descentralizado y se han hecho difusas, en contraste en relación al sistema educativo que aun nos rige, el cual está centralizado en torno a la escuela y el libro (Martín-Barbero, 2002). 


\section{INTERACCIONES DIGITALES DE LAS SUBJETIVIDADES EN EL ECOSISTEMA COMUNICACIONAL}

Se podría decir que estas nuevas formas de estar en el mundo que resultan de la pertenencia a un ecosistema comunicacional viabilizado a través de las TIC, respetan, en tanto estructura pluralista, las condiciones normativas propuestas por Benhabib (2006): reciprocidad igualitaria, autoadscripción voluntaria y libertad de salida y asociación. Estas normas garantizan que el pluralismo cumpla con los objetivos de diversidad cultural e igualdad democrática, expresados a través de una democracia deliberativa universalista.

Martín-Barbero (2002) señala que la convergencia de la globalización y la revolución tecnológica configuran un nuevo ecosistema de lenguajes y escrituras. Cuestión nada despreciable cuando de educación se trata, ya que supone la adquisición progresiva de nuevas configuraciones mentales y corporales, caracterizadas por articulaciones y superposiciones en las que confluyen lo cognitivo, lo sensorial y lo emotivo, más próximas a una razón situada que a la razón ilustrada de Kant. Es así que la convergencia digital implica una convergencia cultural a través de la comunicación digital interactiva. $Y$ es en esta convergencia digital de saberes que transita en pantallas nómades el nuevo escenario en el que las nuevas generaciones tramitan buena parte de su existencia y, por consiguiente, el tiempo-lugar en el que producen sus subjetividades (Amador, 2010).

Cuando Amador (idem) se refiere a las convergencias culturales, no sólo remite a lo digital y lo técnico, sino al propio sujeto que deviene hiperconectado, que accede a modalidades de comunicación, repertorios culturales y experiencias vitales, donde se enlazan distintas dimensiones, incluida la de su propio cuerpo. Surgen en ese contexto manifestaciones de nuevos lenguajes con sus correspondientes hibridaciones. Surgen saberes que demarcan nuevas formas de gestión de la vida, del orden social y cultural. Saberes procedentes de las nuevas necesidades de interactividad y conectividad, saberes co-construidos, cargados de prácticas colaborativas, cooperativas y distribuidas. Esta convergencia cultural que surge a través de la comunicación digital interactiva, tiene atributos de la comunicación deliberativa referenciada por Benhabib (2006), ya que tiene lugar entre sujetos que deciden de forma autónoma cuándo entrar o salir de esos escenarios digitales donde se recrean permanentemente las fronteras culturales.

$\mathrm{El}$ mundo virtual puede ser visto como un no-lugar que permite estar dentro y fuera a la vez. Permite que el sujeto entre con estas nuevas subjetividades y de alguna forma se negocien los espacios a partir de las fricciones que surgen de la interacción digital comunicativa. Deja de ser un encuentro del tipo de multiculturalismo mosaico, porque las identidades no se configuran como una sumatoria de identidades que conviven, sino que se alteran mutuamente a través de una interacción horizontal. Es un espacio que constituye un marco de homogeneización y de voluntad de borradura de las diferencias, pero donde al mismo tiempo y en cierta medida, las diferencias logran escapar y manifestarse. Es claramente un espacio complejo.

Estos nuevos escenarios de vida de los estudiantes no están siendo considerados en toda su dimensión y riqueza. Según Claro (2010, p. 24), es necesario 
“[...] mirar lo que están aprendiendo los niños y jóvenes como producto del uso informal de las TIC fuera del colegio". Esta autora plantea que

[...] una parte importante de los estudiantes hace un uso más intensivo de las TIC fuera del colegio que dentro de él y por lo tanto las nuevas generaciones están aprendiendo cosas de forma no intencionada que son importantes de estudiar y entender. (idem, ibidem)

Aquí entramos de lleno en la dimensión cultural. Poder estudiar y comprender estos aprendizajes, que devienen en nuevas subjetividades, es lo que se propone como dimensión cultural. Esto es muy relevante para poder entender y utilizar las TIC en su plena potencialidad. Se trata de articular la dimensión pedagógica con su dimensión cultural.

Nos podríamos aventurar a pensar que ignorar esta esencia cultural de las TIC podría conducir a resultados no deseados, por ser esta una dimensión transversal a las otras dimensiones pedagógicas y sociales. $\mathrm{O}$ de forma inversa, su consideración permitiría extraer la máxima potencialidad de las TIC como instrumento cultural al servicio de ciertas funcionalidades específicas, por ejemplo, la inclusión social y el aprendizaje. Por otro lado, siguiendo la misma línea de argumentación, se podría suponer que algunas experiencias que reducen las TIC a instrumentos pedagógicos y no consideran la relación que establecen los sujetos con tales instrumentos o las subjetividades que generan en tal relación, sean uno de los motivos de resultados negativos o neutros en tal incorporación.

Algunos estudios de experiencias hacen referencia a una cierta resistencia, tanto de los docentes como de las instituciones educacionales en las cuales se pretende incorporar las tecnologías. Ante esta situación cabe preguntarse: ¿̇erá que estos docentes o instituciones educacionales son ajenos a esta invitación porque las TIC no representan ni forman parte de su cultura profesional y cotidiana? Por otro lado, distintas experiencias ponen en evidencia una significativa motivación de los estudiantes cuando participan de prácticas de aprendizaje con TIC: ¿será que este fenómeno surge porque las TIC forman parte de sus entornos culturales? Por último, y en relación con los estudios que dan cuenta de experiencias no exitosas de aprendizaje con TIC, podemos plantear el siguiente interrogante: ¿será que los docentes incorporan las TIC como instrumentos apenas funcionales al aprendizaje y desconsideran el diálogo mínimo necesario para dar lugar a las propias experiencias culturales tanto de los docentes como de los estudiantes, al cual hace referencia Dussel?

Probablemente, en muchos casos la dimensión cultural es tomada en cuenta pero de forma secundaria. En este trabajo se propone hacerla emerger como dimensión transversal en cualquier experiencia de aprendizaje con TIC. Las TIC han dejado de ser simplemente un medio, para convertirse en un ecosistema comunicativo que está favoreciendo nuevas formas de estar en el mundo, en el que se generan nuevas subjetividades y nuevas formas de relacionarse. No es neutro formar parte, o no, de este ecosistema comunicacional. Y sería esta necesidad la que se propone explicitar para complementarla y hacerla dialogar con las dimensiones pedagógicas y sociales. 
Todos estos interrogantes forman parte de los cuestionamientos que nos hacemos al intentar hacer emerger la dimensión cultural de las TIC, la menos evidente pero probablemente la más relevante y cuestionadora de las prácticas homogeneizadoras. Incluir esta dimensión significa dar lugar a espacios que valoren la diversidad dentro de prácticas que se derivan de políticas homogeneizadoras. Sería irrumpir desde la externalidad frente a esas políticas, para crear contenidos de diálogos que permitan responder a los problemas planteados desde una comprensión reduccionista e instrumental de las TIC. Explicitar y asumir la dimensión cultural abre espacio a una posible respuesta al título del presente trabajo: ¿es posible valorar la diversidad en el marco de la tendencia homogeneizadora?, siempre que se incorpore la dimensión cultural de las TIC.

\section{REFERENCIAS}

Amador, J. C. Mutaciones de la subjetividad en la comunicación digital interactiva: consideraciones en torno al acontecimiento en los nativos digitales. Signo y Pensamiento, Bogotá: Pontificia Universidad Javeriana, v. 29, n. 57, p. 142-161, jul./dic. 2010. Disponible en: $<$ http://recursostic.javeriana.edu.co/cyl/syp/index.php?option=com_bo oklibrary\&task=view\&id=306\&catid=37\&Itemid=48>. Acceso en: 18 enero 2012.

Bauman, Z. Globalização: as consequências humanas. Rio de Janeiro: Jorge Zahar, 1999. Benнabib, S. Las reivindicaciones de la cultura: igualdad y diversidad en la era global. Buenos Aires: Katz, 2006.

Castells, M.; Tubella, I.; Sancho, T.; Díaz, M.; Wellman, B. Conclusión: cambio tecnológico, inercia histórica y transformación social. In: CAstells, M.; Tubella, I. La transición a la sociedad red en Catalunya. Cataluña: UOC, 2002. p. 419-433. Proyecto Internet Catalunya: la sociedad red en Catalunya. Disponible en: $<$ http://www.uoc.edu/ in3/pic/esp/sociedad_red/informe.html >. Acceso en: 18 enero 2012.

Castoriadis, C. Figuras de lo pensable. Buenos Aires: FCE, 2001.

Claro, M. Impacto de las TIC en los aprendizajes de los estudiantes. Estado del arte. Santiago de Chile: CEPAL, 2010 (Colección Documentos de Proyectos). Disponible en: <http://www.eclac.org/publicaciones/xml/7/40947/dp-impacto-tics-aprendizaje. pdf $>$. Acceso en: 18 enero 2012.

Dussel, E. Transmodernidad e interculturalidad (interpretación desde de la filosofía de la liberación). Asociación de Filosofía y Liberación, México: AFYL, 2004. Disponible en: <http://www.afyl.org/articulos.html >. Acceso en: 18 enero 2012.

.1492: el encubrimiento del otro: hacia el origen del mito de la modernidad. La Paz, Bolivia: Plural Editores, 1994.

Dussel, I.; Quevedo, L. A. Educación y nuevas tecnologías: los desafíos pedagógicos ante el mundo digital. In: Foro Latinoamericano de Educación, 6., 2010, Buenos Aires. Documento Básico... Buenos Aires, Santillana, jun. 2010. 80p. Disponible en: $<$ http://www.oei.es/noticias/spip.php?article6965\&debut_5ultimasOEI=5>. Acceso en: 18 enero 2012. 
Eliot, T. S. A media voz. El primer coro de la roca. Traducción Jorge Luis Borges. [s.1.: s.n.], 1934. Disponible en: <http://amediavoz.com/eliot.htm〉. Acceso en: 30 agosto 2012. Ferrer, G.; Arregui, P. Las pruebas internacionales de aprendizaje en América Latina y su impacto en la calidad de la educación. Criterios para futuras aplicaciones. Santiago: PREAL, 2003.

Foucault, M. El sujeto y el poder. In: Dreufus, H.; Rabinow, P.; Foucault, M. Más allá del estructuralismo y la hermenéutica. México: UNAM, 1988.

Garcia, M. M. A. Políticas educacionais contemporâneas: tecnologias, imaginários e regimes éticos. Revista Brasileira de Educação, Rio de Janeiro: ANPEd; Campinas: Autores Associados, v. 15, n. 45, p. 445-591, set./dez. 2010. Disponible en: <http:// www.scielo.br/scielo.php?script=sci_arttext\&pid=S1413-24782010000300004\&lng= pt\&nrm=iso >. Acceso en: 18 enero 2012.

Grosfoguel, R. Hacia un pluri-versalismo transmoderno decolonial. Tabula Rasa, Bogotá: Universidad Colegio Mayor de Cundinamarca, n. 9, p. 199-215, jul./dic. 2008. Disponible en: <http://www.scielo.org.co/scielo.php?script=sci_arttext\&pid=S179424892008000200011\&lng=pt\&nrm=iso>. Acceso en: 18 enero 2012.

Marín,J. Dimensão histórica da perspectiva intercultural, educação, Estado e sociedade. In: Garcia, R. L. (Org.). Diálogos cotidianos. Petrópolis: DP e Alii; Rio de Janeiro: FAPERJ, 2010. p. 313-330.

Martín-Barbero, J. Tecnicidades, identidades, alteridades: des-ubicaciones y opacidades de la de comunicaciónn el nuevo siglo. Diálogos de la Comunicación, Perú: Federación Latinoamericana de Facultades de Comunicación Social (FELAFACS), n. 64, p. 8-23, 2002. Disponible en: <http://www.infoamerica.org/documentos_pdf/ martin_barbero1.pdf $>$. Acceso en: 18 enero 2012.

Diversidad cultural y convergencia digital. Revista Científica de Información y Comunicación, n. 5, p. 12-25, 2008. Disponible en: <http://www.icjournal-ojs.org/index. php/IC-Journal/article/view/265/261>. Acceso en: 20 dic. 2014.

Martucceli, D. Existen individuos en el Sur? Santiago de Chile: LOM, 2010.

Morin, E. La cabeza bien puesta. Buenos Aires: Nueva Visión, 2002.

La vía. España: Paidós, 2011.

PNUD. Desarrollo humano en Chile 2006. Las nuevas tecnologías: ¿un salto al futuro? Santiago de Chile, 2006. Disponible en: <http://www.desarrollohumano.cl/ informe-2006/informe-2006-COMPLETO.pdf>. Acceso en: 20 dic. 2014.

Prigogine, I. O fim das certezas: tempo, caos e as leis da natureza. São Paulo: Editora da Universidade Estadual Paulista, 1996.

Ruiz, M.J. G. Excellent educational systems. Presentation of the monographic section. Revista Española de Educación Comparada, Madrid: UNED; Sociedad Española de Educación Comparada, n. 18, p. 11-26, 2011. Disponible en: <http://www.sc.ehu.es/ sfwseec/reec18.htm>. Acceso en: 18 enero 2012.

Santos, M. Por otra globalización. Del pensamiento único a la conciencia universal. Bogotá: CAB, 2004. 
Sunkel, G.; Trucco, D. Nuevas tecnologías de la información y la comunicación para la educación en America Latina: riesgos y oportunidades. Santiago de Chile: División de Desarrollo Social. Comisión Económica para América Latina (CEPAL), 2010. Disponible en: <http://repositorio.cepal.org/bitstream/handle/11362/6174/lc13266. pdf?sequence=1>. Acceso en: 20 dic. 2014.

Zızeк, S. Multiculturalismo o la lógica cultural del capitalismo multinacional. In: ZıZeK, S.; Jameson, F. Estudios culturales. Reflexiones sobre el multiculturalismo. Buenos Aires: Paidós, 1998.

\section{SOBRE LA AUTORA}

Georgina Vivanco es doctoranda en ciencias de la educación por la Universidad de Santiago de Chile (Chile).

E-mail: georgina@floripa.com.br 